\title{
Effects of Computer-Based Documentation Procedures on Health Care Workload Assessment and Resource Allocation: An Example From VA Sleep Medicine Programs
}

\author{
Kathleen F. Sarmiento, MD, MPH; Eilis A. Boudreau, MD, PhD; Connor J. Smith, MS; Bhavika Kaul, MD; Nancy \\ Johnson, RN, MSA, BSN; and Robert L. Folmer, PhD
}

Background: Computer-based documentation (CBD) is used commonly throughout the world to track patient care and clinical workloads. However, if capture of clinical services within the electronic health record (EHR) is not implemented properly, patient care services and workload credit will be inaccurate, which impacts business decisions related to demand for care and resources allocated to meet the demand. Understaffing of medical personnel can contribute to delays in treatment, missed treatments, and workforce turnover.

Objective: To illustrate the impact of CBD procedures on health care workload assessment and resource allocation, this article uses data from the US Department of Veterans Affairs Corporate Data Warehouse to provide examples from the Veterans Health Administration (VHA) sleep medicine programs.

Discussion: Inaccurate CBD led to underreporting of sleep medicine services provided at VHA facilities nationwide and contributed to insufficient allocation of resources and personnel. Recent modifications in CBD protocols (Stop Codes) improved the accuracy of data capture and reporting while providing VHA sleep programs with data they can use to advocate for workforce expansion to meet patient care needs.

Conclusions: Inaccurate CBD of clinical workloads can result in inadequate allocation of health care personnel and resources to meet the needs of patients. Untreated sleep disorders are associated with increased risk of depression, anxiety, impaired neurocognitive functions, cardiovascular disease, motor vehicle accidents, and premature death. Educating health care providers and administrators on the importance of accurate designation of clinical services within the EHR is necessary to facilitate improvements in health care availability and delivery.
Author affiliations are listed at the end of the article. Correspondence: Robert Folmer (robert.folmer@va.gov)

Fed Pract. 2020;37(8):368-374 doi: $10.12788 /$ fp.0023
$\mathrm{H}$ ealth care systems are faced with the challenge of meeting increasing patient care demands with finite resources. ${ }^{1}$ Advocating for additional capital-specifically, human resources-requires compelling data that accurately capture workload credit. When workload is not captured accurately, clinicians may be tasked with providing care to a high volume of patients without appropriate resource allocation. This understaffing can delay care delivery and increase the risk of diagnostic and treatment errors. ${ }^{2}$ Furthermore, workers in understaffed medical facilities are more likely to experience burnout, which leads to high workforce turnover.

Computer based documentation (CBD) is used often in medical practices to track patient care and clinical workload. However, improperly designed and implemented CBD systems can contribute to cumbersome documentation tasks and inaccurate or incomplete data capture. ${ }^{3}$ Conversely, CBD can be a useful tool to capture workload credit and can subsequently facilitate justification for medical staff allocation to meet patient care demands. This article uses our experience with US Department of Veterans Affairs (VA) national sleep medicine programs to illustrate the impact of CBD procedures on health care workload assessment and allocation. Specifically, we examine how appropriate workload capture facilitates growth and improves the efficiency of health care programs.

The VA is the largest integrated health care system in the US, serving 9 million veterans at 1,255 facilities, including $170 \mathrm{VA}$ Medical Centers (VAMCs). ${ }^{4}$ As veterans' demands for VA medical services have outpaced available resources, there have been several media reports of lapses in timely care delivery. ${ }^{5-7}$ These lapses have been due, in part, to insufficient workforce resource allocation within the Veterans Health Administration (VHA) facilities. A 2012 audit of physician staffing levels conducted by the VA Inspector General concluded that the VA did not have an effective staffing methodology to ensure appropriate staffing levels for specialty care services. ${ }^{8}$ The lack of staffing plans and productivity standards limits 
the ability of medical facility officials to make informed business decisions regarding the appropriate number of specialty physicians required to meet patient care needs. ${ }^{8}$ In 2017, the Government Accountability Office (GAO) issued a report to Congress that stated the "VA's productivity metrics and efficiency models do not provide complete and accurate information, they may misrepresent the true level of productivity and efficiency across VAMCs and limit the VA's ability to determine the extent to which its resources are being used effectively." 9 To understand how and why many VA medical facilities remain understaffed, and therefore struggle to provide health care to veterans in a timely fashion, a description of VA CBD procedures is provided.

\section{BACKGROUND}

VA Directive 1082 on Patient Care Data requires the capture of all outpatient and inpatient billable encounter data. ${ }^{10}$ Accurate capture of workload informs budget allocation models and is necessary for health care provider (HCP) productivity metrics. These data points help identify staff shortages relative to the generated workload. The Veterans Equitable Resource Allocation (VERA) model is used to allocate general purpose funds to the Veterans Integrated Service Networks (VISNs) regional network of VHA facilities. The underlying data components of the VERA model rely on comprehensive data systems that track and analyze the many management information systems used in VHA. Historically, at least $90 \%$ of the funds allocated by the VERA model have been attributed directly to patient care. All workload that is appropriately documented is accounted for in the VERA patient classification process, which is the official data source for funding patient care in VHA.

VA medical facilities use Stop Codes (formerly known as Decision Support System Identifiers) to identify workload for all outpatient encounters and inpatient professional services. Each code is composed

\begin{tabular}{ll}
\multicolumn{2}{c}{ TABLE Veterans Health Administration Stop Codes } \\
Stop Codes & Descriptions \\
\hline 116 & Respiratory therapy \\
117 & Nursing \\
189 & Store-and-forward telehealth from the veteran's home \\
324 & Telephone medicine \\
349 & Sleep medicine \\
$694,695,696$ & Asynchronous telehealth between facilities
\end{tabular}

of a 6-character descriptor that includes a primary Stop Code and a credit (secondary) Stop Code. Primary Stop Codes-the first 3 numbers in the sequence-designate the main clinical group responsible for patient care, such as sleep medicine or neurology. Secondary Stop Codes-the last 3 numbers in the sequence-further define the primary workgroup, such as the type of services provided (eg, telehealth) or the type of HCP (eg, nurse practitioner). These codes help ensure that workload and generated revenue are allocated or credited to the proper specialty care service. ${ }^{11}$ An example of how changes or inaccuracies in Stop Code reporting can affect VHA clinical workload assessment and resource allocation is provided by the VHA sleep medicine program. 
FIGURE 2 Reported CPAP Consults in VA Sleep Clinics ${ }^{a}$

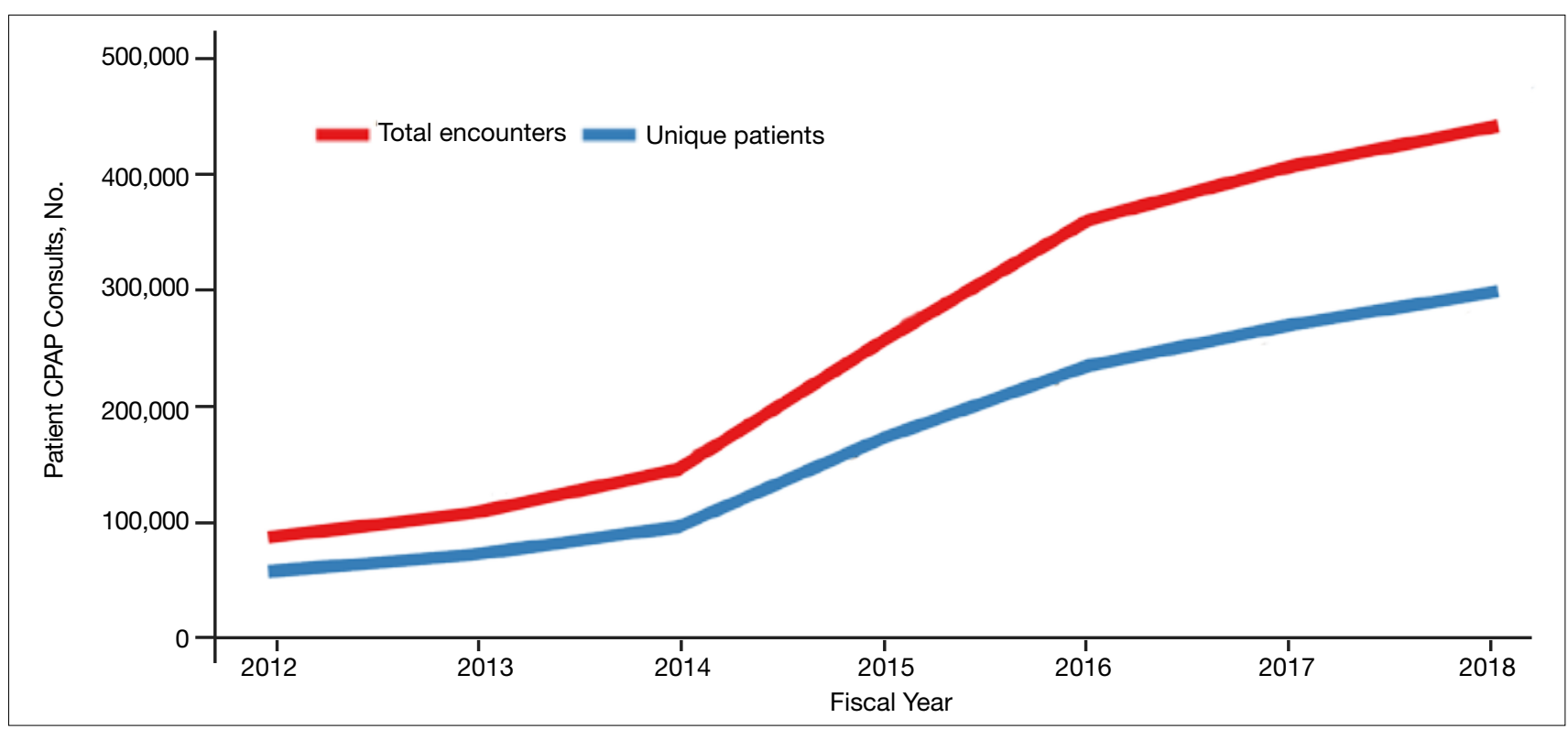

Abbreviations: CPAP, continuous positive airway pressure; VA, US Department of Veterans Affairs.

aStop Codes 349/116 were used to identify these encounters beginning in FY 2015.

The prevalence of sleep disordersparticularly apnea and insomnia-among US military service members and veterans has increased dramatically over the past 2 decades and continues to rise. ${ }^{12-14}$ Consequently, demand for sleep care services at VHA facilities also has increased substantially (Figure 1). Unfortunately, this demand has outpaced the VHA's staffing models, sometimes resulting in long wait times for appointments. ${ }^{15}$ In fact, sleep medicine remains one of the most backlogged services in the VHA, despite significant improvements in program efficiency achieved by incorporating telehealth modalities. ${ }^{16}$ Untreated sleep disorders are associated with increased risk of depression, anxiety, impaired neurocognitive functions, cardiovascular disease, motor vehicle accidents, and premature death. ${ }^{17-23}$

A major contributor to understaffing of VHA sleep medicine programs is the CBD system's historical inability to accurately track sleep resources and demand for sleep care services. For many years, Stop Codes attributed sleep workload credit primarily to pulmonary medicine, neurology, and internal medicine workgroups. Within these workgroups, few individuals contributed to sleep care, but the entire workgroup received credit for these ser- vices, masking the workload of sleep care providers. Additional barriers to accurate sleep medicine workload capture within the VHA included (1) inability to centrally identify personnel, including physicians, as providers of sleep care; (2) limited and variable understanding among VA sleep physicians of the importance of proper encounter form completion (the mechanism by which the cost of a service is calculated); and (3) a lack of awareness that encounter closure is directly linked to productivity measures such as relative value units (RVUs) that support sleep medicine programs and the salaries of those who provide care.

\section{METHODS}

The critical role of accurate CBD in health care administration is illustrated by the proper use of Stop Codes as a foundational step in tracking services provided to justify adequate resource allocation within VA. A complete redesign of tracking sleep service documentation was initiated in 2014 and resulted in national changes to sleep medicine Stop Codes. The Stop Code initiative was the first step of several to improve CBD for VA sleep services.

Primary Stop Code 349 designates sleep medicine encounters in VA facilities (Table). 
FIGURE 3 Reported Telephone Consults in VA Sleep Clinics ${ }^{a}$

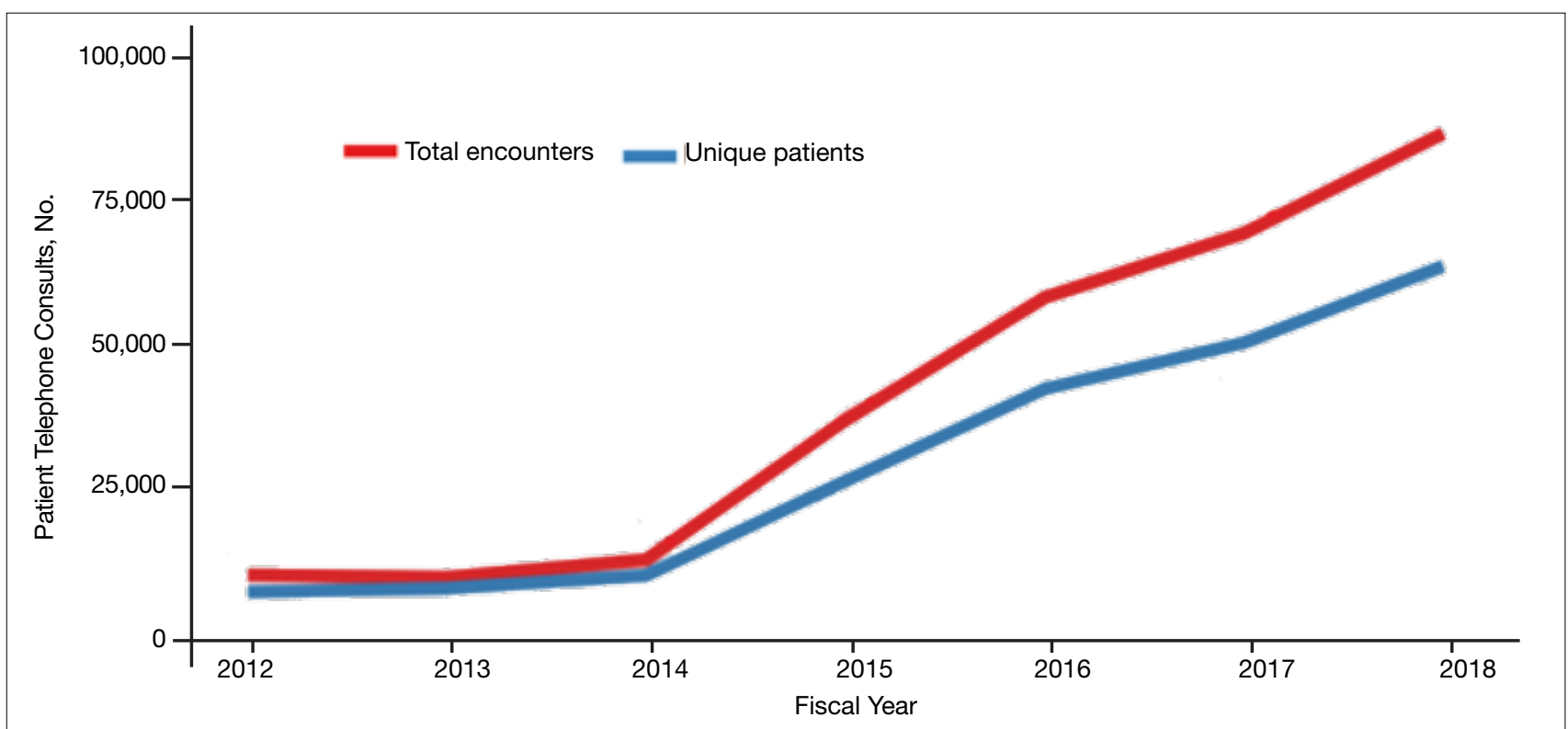

Abbreviation: VA, US Department of Veterans Affairs.

aStop Codes 324/349 were used to identify these encounters beginning in FY 2015.

However, before changes were implemented in fiscal year (FY) 2015, Stop Codes for VHA sleep care did not differentiate between specific services provided, such as laboratorybased sleep testing, at-home sleep testing, education/training sessions, follow-up appointments, equipment consults, telephone or video consults, or administrative tasks. In early FY 2015, several changes were made to Stop Codes used for VHA sleep medicine services nationwide to capture the breadth of services that were being provided; services that had previously been performed but were not documented. A new standardized coding methodology was established for continuous positive airway pressure (CPAP) clinics (349/116 or 349/117); telephone consults for sleep care (324/349); and store and forward sleep telehealth encounters (349/694, $349 / 695$, or 349/696).

In the VA, store-and-forward telehealth refers to asynchronous telemedicine involving the acquisition and storing of clinical information (eg, data, image, sound, or video) that another site or clinician reviews later for evaluation and interpretation. In sleep medicine, data uploaded from home sleep apnea test units or CPAP devices are examples of this asynchronous telehealth model. The goal of these changes in VA Stop Codes was to accurately assess the volume of sleep care delivered and the demand for sleep care (consult volumes); enable planning for resource allocation and utilization appropriately; provide veterans with consistent access to sleep services across the country; and facilitate reductions in wait times for sleep care appointments. Results of these changes were immediate and dramatic in terms of data capture and reporting.

\section{RESULTS}

Figure 1 illustrates an increase in patient encounters in VA sleep clinics by 24,197 (19.6\%) in the first quarter of Stop Code change implementation (FY 2015, quarter 2) compared with those of the previous quarter. VHA sleep clinic patient encounters increased in subsequent quarters of FY 2015 by $29,910(20.2 \%)$ and $11,206(6.3 \%)$ respectively. By the end of FY 2015, reported sleep clinic encounters increased by 190,803 compared with the those at the end of FY 2014, an increase of $42.7 \%$.

Figures 2, 3, and 4 show the additional effects of sleep Stop Code changes that were implemented in FY 2015 for CPAP clinics, telephone encounters, and store-and-forward telehealth encounters, respectively. The large increases in re- 
FIGURE 4 Reported Store-and-Forward Telehealth Encounters in VA Sleep Clinics ${ }^{a-b}$

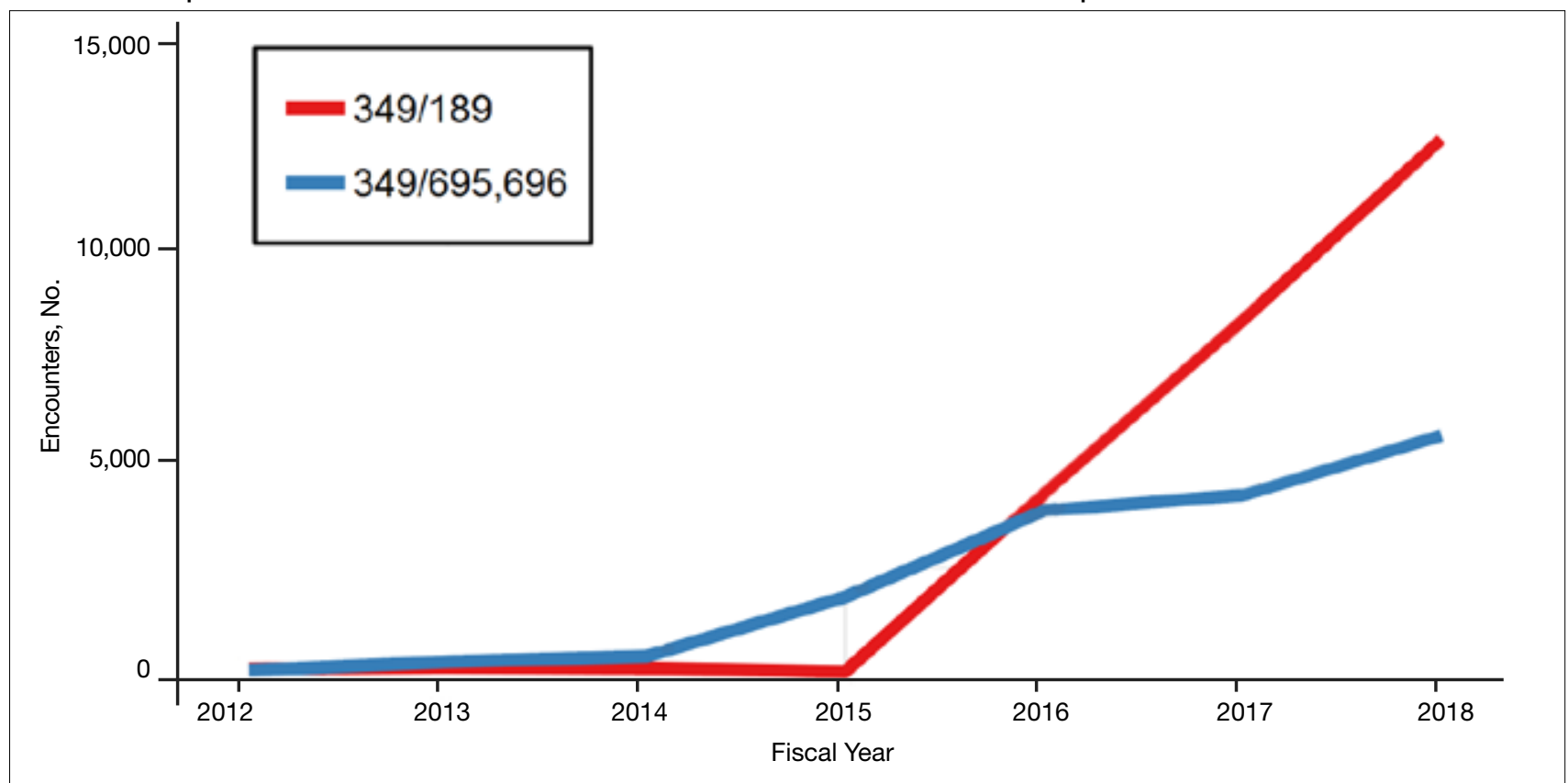

Abbreviation: VA, US Department of Veterans Affairs.

aPrimary Stop Code 349 = sleep medicine.

bSecondary Stop Code 189 = store-and-forward telehealth from the veteran's home.

'Secondary Stop Codes 695 and 696 = store-and-forward telehealth between VA facilities.

ported sleep patient encounters between FY 2014 and FY 2016 reflect changes in CBD and are not entirely due to actual changes in clinical workloads. These results indicate that workloads in many VHA sleep medicine clinics were grossly underreported or misallocated to other specialty services prior to the changes implemented in FY 2015. This discrepancy in care delivery vs workload capture is a contributing factor to the understaffing that continues to challenge VHA sleep programs. However, the improved accuracy of workload reporting that resulted from Stop Code modifications has resulted in only a small proportional increase in VHA clinical resources allocated to provide adequate services and care for veterans with sleep disorders.

In response to the substantial and increasing demand for sleep services by veterans, the VA Office of Rural Health (ORH) funded an enterprise-wide initiative (EWI) to develop and implement a national TeleSleep Program. ${ }^{16}$ The goal of this program is to improve the health and well-being of rural veterans by increasing their access to sleep care and services.

\section{DISCUSSION}

Inaccuracies in CBD procedures can adversely affect health care workload assessment and allocation, contributing to ongoing challenges faced by sleep medicine clinics and other VHA programs that have limited staff yet strive to provide timely and highquality care to veterans. "Not only does inaccurate coding contribute to miscalculations in staffing and resource allocation, it can also contribute to inaccuracies in overall measures of VA healthcare efficiency," the GAO reported to Congress. ${ }^{9}$ The GAO went on to recommend that the VA should ensure the accuracy of underlying staffing and workload data. VHA sleep medicine programs have made efforts to educate HCPs and administrators on the importance of accurate CBD as a tool for accurate data capture that is necessary to facilitate improvements in health care availability and delivery.

In 2018, the VA Sleep Program Office released an updated set of Stop Code changes, including expansion of telehealth codes and improved designation of laboratory and home sleep testing services. These changes are anticipated to result in 
accurate documentation of VA sleep clinic workload and services, especially as the VA TeleSleep EWI to reach rural veterans expands. ${ }^{16}$ In light of the improved accuracy of reporting of delivered sleep services due to changes in Stop Codes over the past 4 years, VHA sleep medicine providers continue to advocate for allocation of resources commensurate with their clinical workload. An appropriate administrative response to the significant clinical workload performed by disproportionately few providers should include the authorization of increased resources and personnel for sleep medicine as well as providing the tools needed to further streamline workflow efficiency (eg, artificial intelligence, machine learning, and population health management).

\section{CONCLUSIONS}

Despite the barriers faced by many large integrated health care systems, VHA sleep medicine leadership continues to implement changes in CBD protocols that improve the accuracy of clinical workload tracking and reporting. Ultimately, these changes will support proposals for increased resources necessary to improve the quality and availability of sleep care for veterans. This example from VA illustrates the importance of accurate workload capture and its role in informing administrators of health care systems as they strive to meet the needs of patients. Although some VA sleep medicine programs continue to face challenges imposed by systemwide limitations, the ORH TeleSleep Program is a major initiative that improves veterans' access to care by disseminating and implementing effective telehealth technologies and strategies. ${ }^{16}$

\section{Acknowledgments}

This work was supported by a VA Office of Rural Health Enterprise-Wide Initiative.

\section{Author affiliations}

Kathleen Sarmiento is the National VHA TeleSleep Lead and Bhavika Kaul is a Research Fellow, both at the San Francisco VA Healthcare System in California. Eilis Boudreau is a Neurologist, and Robert Folmer is a Research Investigator, both at VA Portland Healthcare system in Oregon. Connor Smith is an Informatics Research Associate, Eilis Boudreau is an Associate Professor of Neurology, and Robert Folmer is an Associate Professor of Otolaryngology, all at Oregon Health \& Science University in Portland. Nancy Johnson is the Lead Clinical Analyst, Systems Design and Standardization in the Managerial Cost Accounting Office, VHA Office of Finance. Kathleen Sarmiento is an Associate Professor of Medicine, and Bhavika Kaul is a Critical
Care Medicine Fellow, both at the University of California, San Francisco.

\section{Author disclosures}

The authors report no actual or potential conflicts of interest with regard to this article.

\section{Disclaimer}

The opinions expressed herein are those of the authors and do not necessarily reflect those of Federal Practitioner, Frontline Medical Communications Inc., the US Government, or any of its agencies.

\section{References}

1. World Health Organization. Workload indicators of staffing need (WISN). https://www.who.int/hrh/resources/WISN Eng_UsersManual.pdf?ua=1. Published December 2015. Accessed June 24, 2020.

2. American Association for Respiratory Care. Position statement: best practices in respiratory care productivity and staffing. https://www.aarc.org/wp-content/ uploads/2017/03/statement-of-best-practices_productivity-and-staffing.pdf. Revised July 2015. Accessed June 24, 2020.

3. Wu DTY, Smart N, Ciemins EL, Lanham HJ, Lindberg $\mathrm{C}$, Zheng $\mathrm{K}$. Using EHR audit trail logs to analyze clinical workflow: a case study from community-based ambulatory clinics. AMIA Annu Symp Proc. 2018;2017:1820-1827. Published 2018 Apr 16.

4. US Department of Veterans Affairs, Veterans Health Administration. https://www.va.gov/health.

5. Cohen T. VA crisis: solutions exist, but haven't happened, panel hears. https://www.cnn.com/2014/06/12/politics/ va-reforms/index.html. Published June 12, 2014. Accessed June 24, 2020.

6. Richardson B. IG probes uncover more problems at VA hospitals. https://thehill.com/policy/defense/258652-igprobes-uncover-more-problems-at-va-hospitals. Published October 30, 2015. Accessed June 24, 2020.

7. Slack D. Inaccurate VA wait times prelude thousands of vets from getting outside care, probe finds. USA Today. March 3, 2017. https://www.usatoday.com/story/news/ politics/2017/03/03/veterans-affairs-inspector-generalwidespread-inaccuracies-wait-times/98693856. Accessed June 24, 2020.

8. US Department of Veterans Affairs, Office of the Inspector General. Veterans Health Administration: audit of physician staffing levels for specialty care services. https://www. va.gov/oig/pubs/VAOIG-11-01827-36.pdf. Published December 27, 2012. Accessed June 24, 2020.

9. Government Accountability Office. VA health care: improvements needed in data and monitoring of clinical productivity and efficiency. https://www.gao.gov/ assets/690/684869.pdf. Published May 2017. Accessed June 24, 2020.

10. US Department of Veterans Affairs, Veterans Health Administration. VHA Directive 1082. Patient care data capture. https://www.va.gov/vhapublications/ViewPublication. asp?pub_ID=3091. Published March 24, 2015. Accessed June $24,2020$.

11. US Department of Veterans Affairs, Veterans Health Administration. VHA Handbook 1006.02. VHA site classifications and definitions. https://www.va.gov/vhapublications/ViewPublication.asp?pub ID=2970. Published December 30, 2013. Accessed June 24, 2020.

12. Alexander M, Ray MA, Hébert JR, et al. The National Veteran Sleep Disorder Study: Descriptive Epidemiology and Secular Trends, 2000-2010. Sleep. 2016;39(7):1399-1410. Published 2016 Jul 1. doi:10.5665/sleep.5972.

13. A Caldwell J, Knapik JJ, Lieberman HR. Trends and factors associated with insomnia and sleep apnea in all United States military service members from 2005 to 2014. J Sleep Res. 2017;26(5):665-670. doi:10.1111/jsr.12543

14. Klingaman EA, Brownlow JA, Boland EM, Mosti C, Geh- 
rman PR. Prevalence, predictors and correlates of insomnia in US army soldiers. J Sleep Res. 2018;27(3):e12612. doi:10.1111/jsr.12612

15. Sharafkhaneh A, Richardson P, Hirshkowitz M. Sleep apnea in a high risk population: a study of Veterans Health Administration beneficiaries. Sleep Med. 2004;5(4):345350. doi:10.1016/j.sleep.2004.01.019.

16. Sarmiento KF, Folmer RL, Stepnowsky CJ, et al. National Expansion of Sleep Telemedicine for Veterans: The TeleSleep Program. J Clin Sleep Med. 2019;15(9):13551364. doi:10.5664/jcsm.7934

17. Van Dongen HP, Maislin G, Mullington JM, Dinges DF. The cumulative cost of additional wakefulness: dose-response effects on neurobehavioral functions and sleep physiology from chronic sleep restriction and total sleep deprivation [published correction appears in Sleep. 2004 Jun 15;27(4):600]. Sleep. 2003;26(2):117-126. doi:10.1093/ sleep/26.2.117

18. Johnson EO, Roth T, Breslau N. The association of insomnia with anxiety disorders and depression: exploration of the direction of risk. J Psychiatr Res. 2006;40(8):700-708. doi:10.1016/j.jpsychires.2006.07.008
19. Léger D, Bayon V, Ohayon MM, et al. Insomnia and accidents: cross-sectional study (EQUINOX) on sleep-related home, work and car accidents in 5293 subjects with insomnia from 10 countries. J Sleep Res. 2014;23(2):143152. doi:10.1111/jsr.12104

20. Franklin KA, Lindberg E. Obstructive sleep apnea is a common disorder in the population-a review on the epidemiology of sleep apnea. J Thorac Dis. 2015;7(8):1311-1322. doi:10.3978/j.issn.2072-1439.2015.06.11

21. Javaheri S, Redline S. Insomnia and Risk of Cardiovascular Disease. Chest. 2017;152(2):435-444. doi:10.1016/j. chest.2017.01.026

22. Linz D, McEvoy RD, Cowie MR, et al. Associations of obstructivesSleepaApnea with atrial fibrillation and continuous positive airway pressure treatment: a review. JAMA Cardiol. 2018;3(6):532-540. doi:10.1001/jamacardio.2018.0095

23. Ogilvie RP, Lakshminarayan K, Iber C, Patel SR, Lutsey PL. Joint effects of OSA and self-reported sleepiness on incident CHD and stroke. Sleep Med. 2018;44:32-37. doi:10.1016/j.sleep.2018.01.004 